\section{SOME BLOOD CHANGES IN ANKYLOSTOMIASIS.}

BY A. HOCART BREHAUT, B.A., M.D., B.C. CANTAB., LATE RESIDENT MEDICAL OFFICER AT KASR-EL-AINI HOSPITAL, CAIRO.

THAT the anæmia in cases of ankylostomiasis is ane to the presence of a toxin is now generally acknowledged, and it appears probable that the eosinophilia is, in a way, a measure of the amount of toxins circulating in the blood-the function of the eosinophiles is probably the formation of an antitoxin. While in Egypt I made a number of differential counts on adult natives who were suffering from the disease, and as the results, especially as regards the eosinophiles, differ from the European ones, appended is a list of 40 Guernsey. which may be of interest. In the accompanying list 300,400 , or 500 leucocytes were counted.

An analysis of these counts shows that the average percentage of the eosinophiles is small compared to the European percentages, and also that there are none of those high eosinophilic counts which have so frequently been described. The average percentage is only 10 per cent. and the highest individual count is 36.75 per cent. Is there any possible explanation of this? Most of, if not all, the poorer Egyptians suffer from old-standing bilharziosis and this is known to be associated with an eosinophilia. It appears possible, and in fact probable, that they are in a state of tolerance to toxins of a certain nature; and consequently in case of an increase in the amount of toxins circulating in the blood the reaction -i.e., the eosinophilic change-would not be so great as in those who had never suffered from a previous toxæmia of a milar nature.

\begin{tabular}{|c|c|c|c|c|c|c|c|c|c|c|}
\hline Number. & $\begin{array}{c}\text { Red blood } \\
\text { corpuscles per } \\
\text { cubic } \\
\text { millimetre. }\end{array}$ & $\begin{array}{c}\text { White blood } \\
\text { corpuseles per } \\
\text { cubic } \\
\text { millimetre. }\end{array}$ & $\begin{array}{l}\text { Hæmo- } \\
\text { globin. }\end{array}$ & $\begin{array}{l}\text { Colour } \\
\text { index. }\end{array}$ & $\begin{array}{c}\text { Poly- } \\
\text { morpho- } \\
\text { nuclear } \\
\text { leucocytes. }\end{array}$ & $\begin{array}{c}\text { Large } \\
\text { hyaline. }\end{array}$ & $\begin{array}{l}\text { Lympho- } \\
\text { cytes. }\end{array}$ & $\begin{array}{l}\text { Eosino- } \\
\text { philes. }\end{array}$ & Myelocytes. & Mast cells. \\
\hline 1 & $3,208,000$ & 5,600 & 45 & 0.7 & $83 \cdot 00$ & 5 & 7 & 5 & - & - \\
\hline 2 & $2,300,000$ & 28,125 & 32 & 0.69 & $38 \cdot 75$ & $7 \cdot 5$ & $15 \cdot 5$ & $36 \cdot 75$ & - & 0.75 \\
\hline 3 & $2,200,000$ & 9,200 & 24 & 0.545 & $74 \cdot 4$ & $2 \cdot 8$ & $17 \cdot 2$ & 4 & 0.4 & $1 \cdot 0$ \\
\hline 4 & $3,755,200$ & 12,600 & 48 & 0.64 & $63 \cdot 6$ & 2 & 26 & 8 & - & - \\
\hline$: 5$ & 892,000 & 9,208 & $\begin{array}{c}\text { About } \\
8\end{array}$ & 0.44 & $81 \cdot 5$ & 2.5 & $13 \cdot 5$ & $2 \cdot 5$ & - & - \\
\hline 6 & $1,025,000$ & 10,200 & 9 & 0.45 & $67 \cdot 25$ & $2: 25$ & $10 \cdot 25$ & 14 & 6 & - \\
\hline 7 & $2,020,000$ & 12,200 & 20 & 0.5 & 68.75 & 4 & $22 \cdot 25$ & 8 & - & 0.25 \\
\hline 8 & $2,468,000$ & 12,600 & 30 & 0.62 & 68.4 & $3 \cdot 4$ & $17 \cdot 6$ & 10 & 0.2 & $0: 4$ \\
\hline 9 & $1,700,000$ & 12,187 & 12 & 0.35 & $67 \cdot 5$ & 4.5 & $15 \cdot 4$ & 10.5 & 16 & 06 \\
\hline 10 & $2,345,000$ & 12,187 & 29 & 0.62 & $71 \cdot 2$ & 4 & $12 \cdot 8$ & $10 \cdot 1$ & 0.3 & $0 \cdot 7$ \\
\hline 11 & $3,175,000$ & 8,400 & 40 & 0.64 & 59.6 & $3 \cdot 6$ & 24 & $12 \cdot 4$ & $0 \cdot 2$ & 02 \\
\hline 12 & $2,844,000$ & 17,600 & 31 & 0.62 & $77 \cdot 2$ & $6 \cdot 4$ & $10 \cdot 8$ & 2 & - & - \\
\hline 13 & $1,597,500$ & 6,289 & 15 & 0.5 & $71 \cdot 4$ & $3 \cdot 6$ & $17 \cdot 6$ & 8 & 0.4 & - \\
\hline 14 & $1,252,000$ & 18,280 & 12 & 0.5 & $60 \cdot 5$ & 5 & $23 \cdot 5$ & 150 & 0.5 & 0.5 \\
\hline 15 & $1,226,000$ & 9,200 & 14 & 0.58 & $66 \cdot 4$ & 4 & 20 & $8 \cdot 4$ & 0.4 & 08 \\
\hline 16 & $1,860,000$ & 11,000 & 80 & 0.55 & 60.4 & $4 \cdot 4$ & $23 \cdot 6$ & $8 \cdot 6$ & 1 & 1 \\
\hline 77 & $1,570,000$ & 10,000 & 16 & 0.53 & $58 \cdot 75$ & $4 \cdot 25$ & $23 \cdot 25$ & $10 \cdot 5$ & 0.25 & 0.5 \\
\hline 18 & $4,490,000$ & 10,625 & 88 & 0.99 & $55 \cdot 75$ & $1 \cdot 75$ & $27 \cdot 5$ & $13 \cdot 75$ & 0.5 & 0.75 \\
\hline 79 & $1,551,200$ & 10,800 & 15 & 0.5 & $61 \cdot 00$ & $6 \cdot 2$ & 21 & $11 \cdot 4$ & - & 0.4 \\
\hline 20 & $2,080,000$ & 9,000 & 22 & 0.55 & $79 \cdot 6$ & $3 \cdot 6$ & $14 \cdot 00$ & $2 \cdot 4$ & - & 0.4 \\
\hline 21 & $2,400,000$ & 8,125 & 30 & 0.62 & 72 & 6.5 & $16 \cdot 0$ & $3 \cdot 5$ & - & 0.5 \\
\hline 22 & $1,625,000$ & 10,525 & 20 & 0.62 & 60.5 & $4 \cdot 5$ & 25 & 10 & - & - \\
\hline 23 & $1,638,009$ & 19,200 & 14 & 0.43 & 62 & 6 & $19 \cdot 2$ & $10 \cdot 8$ & 1 & 1 \\
\hline$: 24$ & $2,160,000$ & 10,600 & 20 & 0.46 & 66 & $3 \cdot 2$ & $12 \cdot 8$ & 16 & - & $2 \cdot 0$ \\
\hline 25 & $2,025,000$ & 10,000 & 23 & 0.57 & $68 \cdot 75$ & $3 \cdot 25$ & $21 \cdot 5$ & $6 \cdot 5$ & - & - \\
\hline 26 & $2,960,000$ & 13,200 & 33 & 0.57 & 44.8 & 8 & $29 \cdot 6$ & $16 \cdot 8$ & - & 0.8 \\
\hline 27 & $4,792,000$ & 10,200 & 86 & 0.90 & $67 \cdot 4$ & 3 & $19 \cdot 4$ & $8 \cdot 4$ & 0.8 & - \\
\hline 28 & $4,250,000$ & 9,200 & 65 & 0.77 & $60 \cdot 5$ & 2 & $27 \cdot 5$ & $9 \cdot 5$ & - & 0.5 \\
\hline 29 & $2,038,000$ & 12,800 & 17 & 0.42 & 66.5 & $2 \cdot 5$ & $18 \cdot 5$ & 12 & 0.5 & - \\
\hline 30 & $3,950,000$ & 6,000 & 54 & 0.69 & 61 & $4 \cdot 6$ & 19 & $13 \cdot 8$ & - & $1 \cdot 6$ \\
\hline 31 & $1,500,000$ & 10,200 & 16 & 0.53 & $60 \cdot 5$ & 4 & $21 \cdot 25$ & $12 \cdot 25$ & - & 0.5 \\
\hline 32 & $1,512,000$ & 11,200 & 15 & 0.50 & $72 \cdot 8$ & $4 \cdot 4$ & 16 & 6.8 & - & - \\
\hline 33 & $3,883,300$ & 10,600 & 55 & 0.72 & 65 & 4 & 22 & 8 & 0.5 & $0-5$ \\
\hline 34 & $2,600,000$ & 8,000 & 25 & 0.57 & 70 & 7 & 12 & 10.5 & - & 0.5 \\
\hline 35 & $1,062,160$ & 20,600 & 10 & 0.50 & 75 & $3 \cdot 6$ & $18 \cdot 2$ & $2 \cdot 8$ & - & 0.4 \\
\hline 36 & $2,360,000$ & 10,800 & 19 & 0.40 & $60 \cdot 8$ & $5 \cdot 6$ & 26 & $6 \cdot 8$ & - & 0.8 \\
\hline 37 & $1,962,000$ & 19,062 & 22 & 0.57 & $76 \cdot 5$ & $2 \cdot 5$ & 17 & $2 \cdot 5$ & - & $1 \cdot 5$ \\
\hline 38 & $2,260,000$ & 7,000 & 27 & 0.61 & $65 \cdot 2$ & 4 & 14 & $15 \cdot 4$ & 0.4 & 1.0 \\
\hline 39 & $2,692,600$ & 13,000 & 27 & 0.50 & 60 & $7 \cdot 6$ & 18 & 12 & 1 & $1-2$ \\
\hline 40 & $3,155,200$ & 10,000 & 44 & 0.70 & 66 & $2 \cdot 6$ & 16 & $14 \cdot 4$ & $1 \cdot 0$ & - \\
\hline Total $\ldots$ & $94,383,860$ & 465,613 & 1152 & $23 \cdot 165$ & $2646 \cdot 2$ & $169 \cdot 6$ & $751 \cdot 7$ & $400 \cdot 05$ & $16 \cdot 95$ & $20 \cdot 05$ \\
\hline Average... & $2,359,596$ & 11,640 & $28 \cdot 8$ & 0.59 & $66 \cdot 15$ & $4 \cdot 24$ & $18 \cdot 79$ & $10 \cdot 00$ & $0 \cdot 42$ & 0.50 \\
\hline
\end{tabular}

1. Only seven ankylostoma worms were present. 2. This was the highest eosinophile count found. There was also a large increase in the leucocyte count. 3. Unusually large number of small nucleated red cells were present. 4. 0.4 transitional. Many nucleated red cells were seen.
5. It was impossible to estimate accurately the hæmoglobin as it was so small. Extreme variation in size and shape of 16 cells. Very numerous 5. It was impossible to estimate accurately the hæmoglobin as it was so small. Extreme variation in size and shape of led cells. Very numerous
blood platelets. Fatal case. 6. Fatal case. There was marked variation in size and shape of red cells. Platelets rery abundant. 10 . 0.9 transitional. blood platelets. Fatal case. 6. Fatal case. There was marked variation in size and shape of red cells. Platelets rery abundant. 10. 0.9 transitional.
12 . A large number of platelets present. Patient had pellagra and recent and old bilharziosis. 13. Many nucleated red cells present. Much alteration
in size of the non-nucleated ref cells. 15. Marked tentency to degeneration in the leucocytes. 16. 1 per cent. transitional. 17. Six transitional in size of the non-nucleater re cells. 15. Marked tenclency to degeneration in the leucocytes. 16. 1 per cent. transitional. 17. Six transitional cells were counteil in 403. 18. A mild case. 12 worms only ; no bilharzia present. 19. One transitional cell seen. 21. Two transitional cells in 700.
Platelets abundant. Fatal case. $39.0 \cdot 4$ transitional. 